\title{
Is Chikungunya Virus Infection an Independent Risk Factor for 2-Year Mortality in Older Afro-Caribbean Subjects?
}

This article was published in the following Dove Press journal: Infection and Drug Resistance

\author{
Lidvine Godaert ${ }^{\prime}$ \\ Camille Nicolon ${ }^{2}$ \\ Fatiha Najioullah ${ }^{3}$ \\ Lukshe Kanagaratnam ${ }^{4}$ \\ Raymond Césaire ${ }^{3,5}$ \\ Moustapha Dramé (iD $)^{5,6}$ \\ 'Department of Geriatrics, General \\ Hospital of Valenciennes, Valenciennes, \\ France; ${ }^{2}$ Department of Geriatrics, \\ Hospital of Marennes, Marennes, France; \\ ${ }^{3}$ Department of Virology, University \\ Hospitals of Martinique, Fort-de-France, \\ Martinique; ${ }^{4}$ Department of Research \\ and Public Health, University Hospitals of \\ Reims, Reims, France; ${ }^{5}$ Faculty of \\ Medicine, University of French West \\ Indies, Fort-de-France, Martinique; \\ ${ }^{6}$ Department of Research and Innovation, \\ University Hospitals of Martinique, Fort- \\ de-France, Martinique
}

Purpose: The aim of this study was to investigate whether Chikungunya virus infection (CVI) was an independent risk factor for 2-year mortality in Afro-Caribbean subjects aged 65 years or older.

Patients and methods: A retrospective cohort study was performed from January 2014 to December 2016 in the University Hospital of Martinique. Subjects aged $\geq 65$ years admitted to the hospital were included. Baseline characteristics and concurrent manifestations at admission were collected. Subjects were followed up by phone for 2 years.

Results: A total of 687 old Afro-Caribbean subjects (80.4 \pm 8.0 years) were included: 467 positive for CVI (Chik+) and 220 negative for CVI (Chik-). During the follow-up, 180 (26.2\%) died. The proportion of deaths was higher among Chik- $(40.9 \%)$ than among Chik+ subjects $(21.6 \%)(\mathrm{p}<0.0001)$. By multivariable analysis, when adjusted for age polyarthralgia, neurological troubles, cardiovascular disorders, absence of neutrophilia, thrombocytopenia, hypernatremia, and hospital stay, Chik + subjects had significantly higher survival rates (HR: 0.58 ; 95\% CI: $0.40-0.85$ ) than Chik- ones.

Conclusion: Within the two years following hospital admission of subjects aged $\geq 65$ years or older, Chik+ subjects had significantly higher survival rates than Chik- ones.

Keywords: Chikungunya, survival, elderly people, hospital admission

\section{Introduction}

Chikungunya virus infection (CVI) is an arbovirus transmitted by Aedes (Ae) aegypti or Ae albopictus mosquito bites. CVI was long considered as a disease of the tropics and subtropics, but the spread of its vector into more temperate zones, ${ }^{1}$ as well as adaptation of the virus, ${ }^{2}$ and human migration have made CVI an emerging disease in temperate countries. ${ }^{1}$ Godaert et al previously reported that older subjects (aged 65 and older) may have atypical presentation as well as high hospitalisation rates. ${ }^{3}$ During epidemics, an increase in mortality has been described in various regions, ${ }^{4}$ while some fatal CVI cases have been described at the acute phase. ${ }^{5}$ The population of subjects aged 65 or older is at risk of severe forms of the disease at the acute phase. ${ }^{5}$ Godaert et al reported an in-hospital mortality rate of $9.1 \%{ }^{6}$ The reputation of low fatality associated with acute phase CVI is now being called into question. ${ }^{7}$ Even in the more typical forms, CVI is highly debilitating at the acute phase, and may have medium and long-term repercussions, in particular the development of chronic disease. ${ }^{8}$ To the best of our knowledge, the impact of CVI on mortality in the medium to long term is not documented. Age and a
Correspondence: Moustapha Dramé Department of Research and Innovation CS 90632, University Hospitals of Martinique - Pierre Zobda-Quitman Hospital, Fort-de-France 9726I, Martinique Tel +596596559769

Email moustapha.drame@chu-martinique.fr 
previous history of certain diseases have been identified as risk factors for the occurrence of chronic forms of the disease. ${ }^{9,10}$ In this context, older populations could be at higher risk of medium to long-term sequelae after initial infection with the Chikungunya virus. The aim of this study was therefore to investigate whether CVI was an independent risk factor for 2-year mortality in AfroCaribbean subjects aged 65 years or older who had been infected by Chikungunya virus.

\section{Patients and Methods}

\section{Study Design and Subjects}

This was a retrospective cohort study performed in the University Hospitals of Martinique (French West Indies). Eligible subjects were aged 65 years or older, admitted to acute care units including the emergency department (ED), for suspected CVI, from 10 January to 31 December 2014, and who underwent biological testing using Reverse Transcription Polymerase Chain Reaction (RT-PCR). Subjects whose clinical and/or biological data were missing in their medical records were excluded. There was no concurrent circulation of dengue at the time of the inclusions.

\section{Data Collection}

Data were retrospectively collected from medical files. The following baseline characteristics were recorded: age, sex, presence or absence of fever and/or polyarthralgia, and hospital stay. We also recorded any of the following unusual concurrent clinical manifestations at admission: infectious disease (other than Chikungunya virus infection), neurological troubles (including confusion or delirium, seizure, syncope, encephalitis, and sensorimotor deficit), cardiovascular disorders (including rhythm disorder, cardiac decompensation, and myocardial ischemia), decompensated diabetes (diabetes with negative impact on glycemic control), respiratory disorders (including dyspnea, respiratory infection based on clinical examination, or positive sputum gram stain, or culture for bacteria, or simply an infiltrate on chest X-Ray), renal failure, hepatic disorders, lymphopenia (count $<1000 / \mathrm{mm}^{3}$ ), absence of neutrophilia (count $<7500$ / $\mathrm{mm}^{3}$ ), thrombocytopenia (count $<100,000 / \mu \mathrm{L}$ ), hypernatremia (blood sodium level $>145 \mathrm{mmol} / \mathrm{L}$ ). The comorbidity burden was assessed using Charlson's comorbidity index. ${ }^{11}$ All subjects included in this study underwent serum sample testing using RT-PCR with the RealStar ${ }^{\circledR}$ Chikungunya RTPCR Kit (Altona Diagnostics GmbH, Hamburg, Germany), and were classified as Chik+ (for positive RT-PCR) and Chik- (for negative RT-PCR).

\section{Ethical Considerations}

The study was performed in accordance with the Declaration of Helsinki and French legislation relating to research involving human beings. The study was approved by the French National authority for the protection of privacy and personal data (Commission nationale de l'informatique et des libertés, CNIL): authorisation number 1898399 v 0. The Ethics committee of the University Hospital of Martinique approved the study.

\section{Statistical Analyses}

Quantitative variables are described as mean and standard deviation $(\mathrm{m} \pm \mathrm{SD})$, and categorical variables as number and percentage. Bivariable analysis was performed using Cox model. Kaplan-Meier curves were constructed, and the log-rank test was used to test survival differences between CHIK+ and CHIK- subjects.

A multivariable Cox model was constructed to derive the prediction model. Time to two-year mortality was the primary outcome. The proportionality of risks over time was verified graphically. ${ }^{12}$ Survival time was defined as the duration between hospital admission and date of last contact. The vital status was updated by phone and from the hospital network data-processing system. Baseline characteristics were considered as potential confounders for two-year mortality. A manual stepwise method was used to identify variables independently associated with two-year mortality, after checking for confounders and interactions. Results are presented as hazard ratio (HR) and $95 \%$ confidence interval (CI).

Tests were considered significant for a p-value $<0.05$. Statistical analyses were performed using SAS version 9.4 (SAS Institute Inc., Cary, NC, USA). The Kaplan-Meier curves were computed using IBM SPSS software release 21 (IBM Corporation, Armonk, NY, USA).

\section{Results}

A total of 687 subjects (467 Chik+ and 220 Chik-) were included in the study. Mean age was $80.4 \pm 8.0$ years. There was no difference between Chik + and Chik- in terms of comorbidity burden (Charlson score $1.69 \pm 1.94$ versus 1.64 \pm 1.80 , respectively; $\mathrm{p}=0.73$ ). The demographic, clinical and biological characteristics at inclusion of subjects are presented in Table 1. 
Table I Demographic, Clinical and Biological Characteristics at Inclusion of Subjects According to Their RT-PCR results

\begin{tabular}{|c|c|c|c|c|c|c|c|}
\hline \multirow[t]{2}{*}{ Symptoms or Association of Symptoms } & \multicolumn{2}{|c|}{ All $N=687$} & \multicolumn{2}{|c|}{ Chik- $n=220$} & \multicolumn{2}{|c|}{ Chik+ $n=467$} & \multirow[t]{2}{*}{$\mathbf{p}$} \\
\hline & $\mathbf{n}$ & $\%$ & $\mathbf{n}$ & $\%$ & $\mathbf{n}$ & $\%$ & \\
\hline Age $\geq 85$ years & 508 & 73.9 & 155 & 70.5 & 353 & 75.6 & 0.15 \\
\hline Male sex & 339 & 49.3 & 119 & 54.1 & 220 & 47.1 & 0.09 \\
\hline Fever & 502 & 73.1 & $|3|$ & 59.6 & 371 & 79.4 & $<0.0001$ \\
\hline Polyarthralgia & 244 & 35.5 & 38 & 17.3 & 206 & 44.1 & $<0.0001$ \\
\hline Other infectious diseases & 179 & 26.1 & 90 & 40.9 & 89 & 19.1 & $<0.0001$ \\
\hline Neurological troubles & 61 & 8.8 & 33 & 15.0 & 28 & 6.0 & 0.0001 \\
\hline Cardiovascular disorders & 44 & 6.4 & 15 & 6.8 & 29 & 6.2 & 0.76 \\
\hline Decompensated diabetes & 10 & 1.5 & 7 & 3.2 & 3 & 0.6 & 0.009 \\
\hline Respiratory disorders & 18 & 2.6 & II & 5.0 & 7 & 1.5 & 0.007 \\
\hline Renal failure & 278 & 40.5 & 103 & 46.8 & 175 & 37.5 & 0.02 \\
\hline Hepatic disorders & 91 & 13.2 & 50 & 22.7 & 41 & 8.8 & $<0.0001$ \\
\hline Lymphopenia & 407 & 61.3 & 62 & 30.1 & 345 & 75.3 & $<0.0001$ \\
\hline Absence of neutrophilia & 517 & 77.9 & 102 & 49.5 & 415 & 90.6 & $<0.0001$ \\
\hline Thrombocytopenia & 79 & 11.5 & 28 & 12.7 & 51 & 10.9 & 0.49 \\
\hline Hypernatremia & 20 & 2.9 & 17 & 7.7 & 3 & 0.6 & $<0.0001$ \\
\hline Hospital admission & 488 & 73.8 & 162 & 82.6 & 326 & 70.1 & 0.0008 \\
\hline
\end{tabular}

Notes: Missing data (n): lymphopenia (23); absence of neutrophilia (23); hospital admission (26)

Abbreviations: Chik+, laboratory-confirmed positive Chikungunya infection; Chik-, laboratory-confirmed negative Chikungunya infection.

Among the 687 subjects included, 72 were lost to follow-up and $180(26.2 \%)$ died during the 2 years of follow-up. The proportion of deaths was higher among Chik- (40.9\%) than among Chik+ subjects $(21.6 \%$, $\mathrm{p}<0.0001)$. By bivariable analysis, as shown in Table 2 and Figure 1, Chik+ subjects had significantly higher survival rates than Chik- ones $(p<0.0001)$. This difference persists in the same direction even when comparing only severe Chik + subjects to Chik- subjects $(\mathrm{p}<0.0001)$. By multivariable analysis (Table 2), when adjusted for age, polyarthralgia, neurological troubles, cardiovascular disorders, neutropenia, thrombocytopenia, hypernatremia, and hospital admission, Chik + patients still had significantly higher survival rates than Chik- subjects $(p=0.005)$. By sensitivity analysis, when adjusted for the same confounders, severe Chik+ subjects still had lower hazard of death than Chik- subjects (HR: 0.73; 95\% CI: 0.56-0.96; $\mathrm{p}=0.02$ ).

\section{Discussion}

In our study population, overall two-year mortality was $26.2 \%$. This mortality rate is lower than that previously observed in Afro-Caribbean subjects hospitalised through the emergency department (48.4\%), ${ }^{13}$ and also lower than that described by Drame et $\mathrm{al}^{14}$ in the French SAFES cohort (44\%). However, in our population, only $73.8 \%$ of the subjects were hospitalised after their initial management. The in- hospital mortality during CVI has previously been estimated at $9.1 \%$ by Godaert et $\mathrm{al}^{15}$ in a population of subjects aged 65 years and older, and at $10.6 \%$ by Economopoulou et $\mathrm{al}^{5}$ in an adult population with a median age of 70 years [range 15-95]. As shown in Figure 1, mortality appeared to be higher in the first 90 days after the initial acute episode, regardless of the Chikungunya status. In our study, Chik+ subjects had a two-year mortality rate that was significantly lower than that of the Chik- subjects (respectively $21.6 \%$ vs $40.9 \%, \mathrm{p}<0.0001)$. This survival difference persisted over the whole duration of the follow-up period.

By multivariable analysis, we identified six factors that were significantly associated with increased risk of death, and three factors significantly associated with lower risk of death within a two-year follow-up. The risk factors were age $\geq 85$ years, the presence of neurological signs at admission, cardiovascular signs at admission, thrombocytopenia, hypernatremia, and admission to the hospital during the acute episode. Age has previously been reported to be a risk factor for death at three years in elderly ( $\geq 75$ years) subjects, as have neurological signs such as confusion. ${ }^{14}$ Hypernatremia has also been reported to be independently associated with mortality. ${ }^{16,17}$ Heart failure or acute decompensation is also a prognostic factor for long-term mortality. ${ }^{18}$ Hospital stay can be considered as a sign of the severity of the initial clinical status. The factors found in our study to be associated with lower risk of death were 
Table 2 Bivariable and Multivariable Analyses of the Factors Independently Associated with Two-Year Mortality

\begin{tabular}{|c|c|c|c|c|c|c|}
\hline \multirow[t]{2}{*}{ Characteristics } & \multicolumn{3}{|c|}{ Bivariable Analysis } & \multicolumn{3}{|c|}{ Multivariable Analysis } \\
\hline & HR & $95 \% \mathrm{Cl}$ & $\mathbf{p}$ & aHR & $95 \% \mathrm{Cl}$ & $\mathbf{p}$ \\
\hline Age $\geq 85$ years & 1.72 & $1.17-2.53$ & 0.006 & 1.94 & $1.27-2.98$ & 0.002 \\
\hline Male sex & 1.20 & $0.90-1.61$ & 0.21 & & & \\
\hline Chik+ & 0.38 & $0.28-0.51$ & $<0.000$ I & 0.58 & $0.40-0.85$ & 0.005 \\
\hline Fever & 0.59 & $0.44-0.80$ & 0.0008 & & & \\
\hline Polyarthralgia & 0.39 & $0.27-0.56$ & $<0.0001$ & 0.56 & $0.37-0.84$ & 0.005 \\
\hline Other infectious diseases & 1.66 & $1.22-2.60$ & 0.001 & & & \\
\hline Neurological troubles & 1.34 & $0.84-2.13$ & 0.22 & 1.59 & $1.10-2.29$ & 0.01 \\
\hline Cardiovascular disorders & 2.05 & $1.29-3.26$ & 0.003 & 1.79 & $1.10-2.93$ & 0.02 \\
\hline Decompensated diabetes & 1.16 & $0.37-3.64$ & 0.80 & & & \\
\hline Respiratory disorders & 1.59 & $0.75-3.39$ & 0.23 & & & \\
\hline Renal failure & 1.46 & $1.10-1.98$ & 0.009 & & & \\
\hline Hepatic disorders & 1.64 & $1.13-2.39$ & 0.009 & & & \\
\hline Lymphopenia & 0.51 & $0.38-0.58$ & $<0.000$ I & & & \\
\hline Absence of neutrophilia & 0.37 & $0.27-0.50$ & $<0.000$ I & 0.50 & $0.34-0.72$ & 0.0003 \\
\hline Thrombocytopenia & 1.92 & $1.32-2.80$ & 0.0007 & 2.14 & $1.40-3.26$ & 0.0004 \\
\hline Hypernatremia & 4.58 & $2.60-8.06$ & $<0.0001$ & 1.96 & $1.00-3.87$ & 0.05 \\
\hline Hospital admission & 1.88 & $1.26-2.81$ & 0.002 & 1.45 & $0.96-2.18$ & 0.08 \\
\hline
\end{tabular}

Abbreviations: HR, crude hazard ratio; aHR, adjusted hazard ratio; $95 \% \mathrm{Cl}, 95 \%$ confidence interval of the hazard ratios; Chik+, laboratory-confirmed positive Chikungunya infection.

the presence of polyarthralgia, the absence of neutrophilia, and laboratory confirmation of CVI. Based on our observations, subjects with laboratory confirmed positive CVI died less often than those who attended the hospital with another type of acute event (whatever the cause). In view

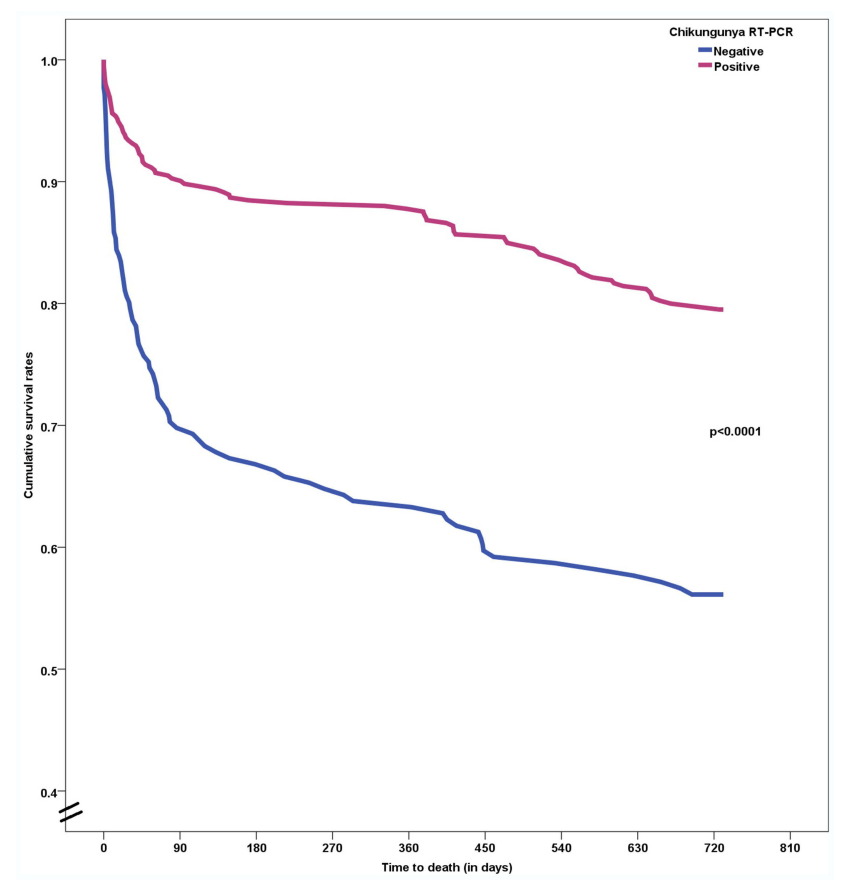

Figure I Kaplan-Meier curves according to the results of the RT-PCR in Chikungunya virus infection. of this finding, we purport that CVI may be a once-off acute health event among these elderly subjects who otherwise would not have consulted the healthcare system at that point of their lives. At the acute phase, the highly debilitating nature of the disease prompts these patients to consult the healthcare system, because they are experiencing acutely and first-hand, a situation of substantial dependence. The more frequent hospitalisation of Chik+ subjects compared to Chik- ones (Table 1) reflects the major impact of the acute CVI phase on their state of health. After the acute phase, subjects with good functional reserve recover well, and CVI does not affect their survival. Some authors have reported excess mortality during the CVI outbreak. ${ }^{19}$ It is a common phenomenon that epidemics lead to immediate excess mortality, especially among the most vulnerable people. ${ }^{20,21}$ But in our article, the aim was not to evaluate the excess mortality related to the CVI outbreak, but to compare the risk of death of elderly people in the two years following their hospitalization, some for CVI, and the others for different acute somatic reasons.

There is no similar study among the adult population, to the best of our knowledge. Therefore, we are unable to compare risk factors for mortality of the elderly subjects in the present analysis with those of adults aged less than 65 years infected with Chikungunya. Indeed, survival alone does not provide any information about the living conditions 
and quality of life of these subjects. In view of the symptom profile at the acute, ${ }^{22}$ and chronic ${ }^{10,23}$ phases, it would be useful to evaluate whether surviving subjects have greater loss of autonomy, greater need for material or human assistance, and what their quality of life is in the longer term. All these elements are of paramount importance in terms of public health during epidemics, particularly in temperate zones where the population of subjects aged 65 years and older is proportionally high.

The strengths of this study include the fact that it is the largest cohort to date of subjects with Chikungunya infections followed up for a period of two years. The inclusion of the Chik- group enabled comparison with subjects of similar profile, recruited in similar conditions, and presenting an acute health event other than CVI. Furthermore, a large variety of data were reliably collected at admission, and the number of missing data was low.

Conversely, some limitations of this study deserve to be underlined. The causes of death at two years were not recorded. Our data come from a hospital cohort only, and therefore, are likely not representative of the overall general population of elderly subjects. Nonetheless, the patterns of healthcare usage in Martinique are different to those observed in western countries. Indeed, there is less recourse to the general practitioner (GP) as the first port of call, due to the shortage of GPs and primary care providers. In this context, emergency services are often used by patients whose illness does not require hospital management, and consulting the emergency department is not a reliable indicator of the gravity of symptoms at the outset.

\section{Conclusion}

Based on our findings, in this population of subjects aged 65 years and older CVI was significantly associated with a higher survival rate. This is important, in view of the expected spread of CVI in the near future, particularly to temperate zones. In view of the symptom profile of CVI at the acute and chronic phases, this study requires further confirmation, particularly focusing on autonomy and quality of life in the medium to long term.

\section{Acknowledgments}

The authors acknowledge Fiona Ecarnot (EA3920, University Hospitals of Besançon, France) for editorial assistance. This research received no specific grant from any funding agency in the public, commercial or not-forprofit sectors.

\section{Disclosure}

No author has any competing interests to declare.

\section{References}

1. Zeller H, Van Bortel W, Sudre B. Chikungunya: its history in Africa and Asia and its spread to new regions in 2013-2014. J Infect Dis. 2016;214(suppl 5):S436-S440. doi:10.1093/infdis/jiw391

2. Powers AM. How Chikungunya virus virology affects its epidemiology and transmission: implications for influencing public health. $J$ Infect Dis. 2016;214(suppl 5):S449-S452. doi:10.1093/infdis/jiw382

3. Godaert L, Najioullah F, Bartholet S, et al. Atypical clinical presentations of acute phase chikungunya virus infection in older adults. $J \mathrm{Am}$ Geriatr Soc. 2017;65(11):2510-2515. doi:10.1111/jgs.15004

4. Renault P, Josseran L, Pierre V. Chikungunya-related fatality rates, mauritius, India, and Reunion Island. Emerg Infect Dis. 2008;14 (8):1327. doi:10.3201/eid1408.080201

5. Economopoulou A, Dominguez M, Helynck B, et al. Atypical Chikungunya virus infections: clinical manifestations, mortality and risk factors for severe disease during the 2005-2006 outbreak on Reunion. Epidemiol Infect. 2009;137(4):534-541. doi:10.1017/ S0950268808001167

6. Godaert L, Bartholet S, Dorleans F, et al. Prognostic factors of inhospital death in elderly patients: a time-to-event analysis of a cohort study in Martinique (French West Indies). BMJ Open. 2018;8(1):e018838. doi:10.1136/bmjopen-2017-018838

7. Couderc T, Lecuit M. Chikungunya virus pathogenesis: from bedside to bench. Antiviral Res. 2015;121:120-131. doi:10.1016/j.antiviral.2015. 07.002

8. Marimoutou C, Vivier E, Oliver M, Boutin JP, Simon F. Morbidity and impaired quality of life 30 months after chikungunya infection: comparative cohort of infected and uninfected French military policemen in Reunion Island. Medicine (Baltimore). 2012;91(4):212-219. doi:10.1097/MD.0b013e318260b604

9. Couturier E, Guillemin F, Mura M, et al. Impaired quality of life after chikungunya virus infection: a 2-year follow-up study. Rheumatology (Oxford). 2012;51(7):1315-1322. doi:10.1093/rheumatology/kes015

10. Moro ML, Grilli E, Corvetta A, et al. Long-term chikungunya infection clinical manifestations after an outbreak in Italy: a prognostic cohort study. J Infect. 2012;65(2):165-172. doi:10.1016/j.jinf.2012.04.005

11. Sundararajan V, Henderson T, Perry C, Muggivan A, Quan H, Ghali WA. New ICD-10 version of the Charlson comorbidity index predicted in-hospital mortality. J Clin Epidemiol. 2004;57(12):12881294. doi:10.1016/j.jclinepi.2004.03.012

12. Kay R. Goodness of fit methods for the proportional hazards regression model: a review. Rev Epidemiol Sante Publique. 1984;32(34):185-198.

13. Godaert L, Godard-Sebillotte C, Allard Saint-Albin L, et al. Selfrated health as a predictor of mid-term and long-term mortality in older Afro-Caribbeans hospitalised via the emergency department. Qual Life Res. 2017.

14. Drame M, Dia PA, Jolly D, et al. [Factors predictive of long-term mortality in patients aged 75 years or older hospitalized from the emergency department: the SAFES cohort]. Presse Med. 2009;38(78):1068-1075. French. doi:10.1016/j.lpm.2009.01.019

15. Godaert L, Bartholet S, Dorléans F, et al. Prognostic factors of inhospital mortality in elderly patients: a time-to-event analysis of a cohort study in Martinique (French West Indies). BMJ Open. 2017.

16. Hu J, Wang Y, Geng X, et al. Dysnatremia is an independent indicator of mortality in hospitalized Patients. Med Sci Monitor. 2017;23:2408-2425. doi:10.12659/MSM.902032

17. Girardeau Y, Jannot AS, Chatellier G, Saint-Jean O. Association between borderline dysnatremia and mortality insight into a new data mining approach. BMC Med Inform Decis Mak. 2017;17 (1):152. doi:10.1186/s12911-017-0549-7 
18. Ruigomez A, Michel A, Martin-Perez M, Garcia Rodriguez LA. Heart failure hospitalization: an important prognostic factor for heart failure re-admission and mortality. Int $J$ Cardiol. 2016;220:855-861. doi:10.1016/j.ijcard.2016.06.080

19. Freitas ARR, Alarcon-Elbal PM, Donalisio MR. Excess mortality in Guadeloupe and Martinique, islands of the French West Indies, during the chikungunya epidemic of 2014. Epidemiol Infect. 2018;146 (16):2059-2065. doi:10.1017/S0950268818002315

20. Lai CC, Wang CY, Wang YH, Hsueh SC, Ko WC, Hsueh PR. Global epidemiology of coronavirus disease 2019 (COVID-19): disease incidence, daily cumulative index, mortality, and their association with country healthcare resources and economic status. Int $J$ Antimicrob Agents. 2020;55(4):105946. doi:10.1016/j.ijantimicag.2020.105946
21. Onder G, Rezza G, Brusaferro S. Case-fatality rate and characteristics of patients dying in relation to COVID-19 in Italy. JAMA. 2020. doi:10.1001/jama.2020.4683

22. Simon F, Javelle E, Cabie A, et al. French guidelines for the management of chikungunya (acute and persistent presentations). November 2014. Med Mal Infect. 2015;45(7):243-263. doi:10.1016/j.medmal. 2015.05.007

23. Schilte C, Staikowsky F, Couderc T, et al. Chikungunya virus-associated long-term arthralgia: a 36-month prospective longitudinal study. PLoS Negl Trop Dis. 2013;7(3):e2137. doi:10.1371/journal. pntd.0002137

\section{Publish your work in this journal}

Infection and Drug Resistance is an international, peer-reviewed openaccess journal that focuses on the optimal treatment of infection (bacterial, fungal and viral) and the development and institution of preventive strategies to minimize the development and spread of resistance. The journal is specifically concerned with the epidemiology of antibiotic resistance and the mechanisms of resistance development and diffusion in both hospitals and the community. The manuscript management system is completely online and includes a very quick and fair peerreview system, which is all easy to use. Visit http://www.dovepress.com/ testimonials.php to read real quotes from published authors. 